\title{
Theoretical Description for Orellanine Electrochemical Determination and Electropolymerization in the Presence of Hydroquinones, Assisted by CuS Nanoparticles
}

\author{
Volodymyr V. Tkach ${ }^{1,2, * \mathbb{D}}$, Marta V. Kushnir ${ }^{1 \mathbb{C}}$, Vira V. Kopiika ${ }^{3 \mathbb{C}}$, Olga V. Luganska ${ }^{3 \mathbb{D}}$, Zholt 0. \\ Kormosh ${ }^{4} \mathbb{D}$, Yevgeniya V. Nazymok ${ }^{5 \mathbb{D}}$, Yana G. Ivanushko ${ }^{5(\mathbb{D})}$, Ruslana V. Slukhenska ${ }^{5(\mathbb{D})}$, \\ Volodymyr D. Moysiuk ${ }^{5}$ (D), Iryna L. Kukovska ${ }^{5(\mathbb{D})}$, Viktor V. Gordiyenko ${ }^{5(\mathbb{D})}$, \\ Mykola Ye. Blazheyevskiy ${ }^{6}(\mathbb{D})$, Karina V. Palamarek ${ }^{7(\mathbb{D})}$, Dina V. Fedorova ${ }^{8}$ (D), \\ Sílvio C. De Oliveira ${ }^{2}$ (D), Galyna M. Pochenchuk ${ }^{1}$ (D), Petro I. Yagodynets ${ }^{1, *}$ (D)
}

1 Chernivtsi National University, 58000, Kotsyubyns'ky Str. 2, Chernivtsi, Ukraine; nightwatcher2401@gmail.com (V.V.T.); ved1988mid@rambler.ru (P.I.Y.); g.pochenchyuk@chnu.edu.ua (G.M.P.); marta.v.kushnir@gmail.com (M.V.K.);

2 Universidade Federal de Mato Grosso do Sul, Av. Sen. Felinto. Müller, 1555, C/P. 549, 79074-460, Campo Grande, MS, Brazil; scolive@gmail.com (S.C.d.O.);

3 Zaporizhzhia National University, 69600, Zhukovsky Str. 66, Zaporizhzhia, Ukraine; 130805olga@ gmail.com (O.V.L.); vkopijka@ukr.net (V.V.K.);

4 Eastern European National University, 43000, Voli Ave., 13, Lutsk, Ukraine; zholt-1971@ukr.net (Z.O.K.);kormosh@eenu.edu.ua (Z.O.K.);

5 Bukovinian State Medical University, 58001, Teatralna Sq., 9, Chernivtsi Ukraine;yana_iv@ukr.net (Y.G.I.); nazymok36@ukr.net (Y.V.N.); kukovska.irina@gmail.com (Y.L.K.); gordienko.viktor@bsmu.edu.ua (V.V.G.); vmoysiuk@gmail.com (V.D.M.); slyruslana@gmail.com (R.V.S.);

6 National Pharmaceutical University, 61000, Pushkinska Str. 57, Kharkiv, Ukraine; blazejowski@ukr.net (M.Y.B.);

7 Chernivtsi Institute of Trade and Economics of Kyiv National University of Trade and Economics, 58012, Central Sq. 9, Chernivtsi, Ukraine; karinkap55@gmail.com (K.V.P.);

8 Kyiv National University of Trade and Economics, 02156, Kyoto Str. 21, Kyiv, Ukraine; dina_fedorova@ ukr.net (D.V.F.);

* Correspondence: nightwatcher2401@gmail.com (V.V.T.); ved1988mid@ rambler.ru (P.I.Y.);

Scopus Author ID 55758299100

Received: 9.10.2020; Revised: 2.11.2020; Accepted: 5.11.2020; Published: 7.11.2020

Abstract: The theoretical description for orellanine electrochemical determination and electropolymerization, assisted by $\mathrm{CuS}$ nanoparticles, deposited over a graphene surface, is given. Taking into account the orellanine similarity to hydroquinonic compounds, alongside with their higher oxidation potential, copper sulfide nanoparticles, paired with copper (III) sulfohydroxide are used. Orellanine polymerization is also promoted, yielding a natural-based conducting polymer with interesting catalytical properties. The analysis of the correspondent mathematical model confirms the efficiency of the system. The linear dependence between the electrochemical parameter and concentration is easy to obtain and maintain. On the other hand, the oscillatory and monotonic instabilities in this system will be more probable than for hydroquinone compound determination.

Keywords: orellanine; food safety; electrochemical sensors, electrochemical polymerization; stable steady-state.

(C) 2020 by the authors. This article is an open-access article distributed under the terms and conditions of the Creative Commons Attribution (CC BY) license (https://creativecommons.org/licenses/by/4.0/).

\section{Introduction}

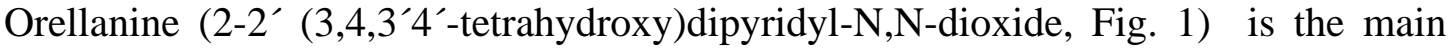
toxin of poisonous mushrooms Orellani (foolish webcaps) [1 - 4]. The first symptoms of 
orellanine poisoning are similar nausea, vomiting, stomach pains, headaches, followed by kidney failure (immense thirst, frequent urination, pain on and around the kidneys). If untreated, death is imminent.

The structure is very similar to that of hydroquinone compounds, and the human receptor will recognize orellanine as one of those, leading to toxic quinone-like forms, participating in radical oxidation processes in the organism, driving it off the normal path. As both the cooked and uncooked mushrooms contain these compounds and as the orellani mushrooms are difficult to be recognized, the orellanine poisoning detection is really actual, and the electrochemical methods, yet used for hydroquinone compounds, may be used for this purpose [5-12].

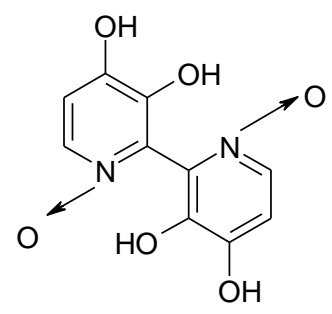

Figure 1. Orellanine structure.

Possessing electroactive groups (N-oxide, aromatic ring, and phenol-like hydroxyls) orellanine is electroactive. Moreover, mimicking the hydroquinone structure, it may be used as a monomer for economical and green (semi)conducting polymer [13 - 19] with interesting catalytic properties. Therefore, the electrochemical (direct or assisted) oxidation of orellanine may serve as the font for both electroanalytical or electrosynthetical purposes.

The modern electroanalytical processes permit the use of chemically modified electrodes, capable of detecting the orellanine and classical hydroquinone compounds similarly. One of the electrode modifiers used for this purpose is nanoparticles [20-24], permitting to detect of a wide range of analytes.

Nonetheless, taking into account the frequency of the electrochemical instabilities observed during the organic molecules electrooxidation and electropolymerization [25-26], capable of influencing directly the electroanalytical system behavior, the process of electrochemical determination and electropolymerization of orellanine in the presence of hydroquinone compounds has to be studied theoretically before it is used in practice. Therefore, the goal of this work is the mechanistic theoretical study of CuS-assisted orellanine and hydroquinone determination by the development and analysis of the corresponding mathematical model. Also, the behavior of the system will be compared to that of similar ones [27-28].

\section{Materials and Methods}

Copper (II) sulfide nanoparticles with the applied anodic potential undergo electrooxidation, yielding copper (III) sulfohydroxide (1):

$$
\mathrm{CuS}+\mathrm{OH}^{-}-\mathrm{e}^{-} \rightarrow \mathrm{CuS}(\mathrm{OH})
$$

It is a highly energetic form, capable of oxidizing both hydroquinones and orellanines. Taking into account the similarity between them, the analytes are oxidized according to a similar mechanism. Nevertheless, orellanines, possessing N-oxidized pyridinic nitrogen atoms, are oxidized more difficultly. Moreover, as orellanines are much more polar, their 
electrooxidation and electro(co)polymerization will affect the double electric layer (DEL) in a more significant manner. Therefore, the behavior of the system will be more dynamic than hydroquinone determination and polymerization. The scheme of the electroanalytical process is exposed as in Fig. 2:

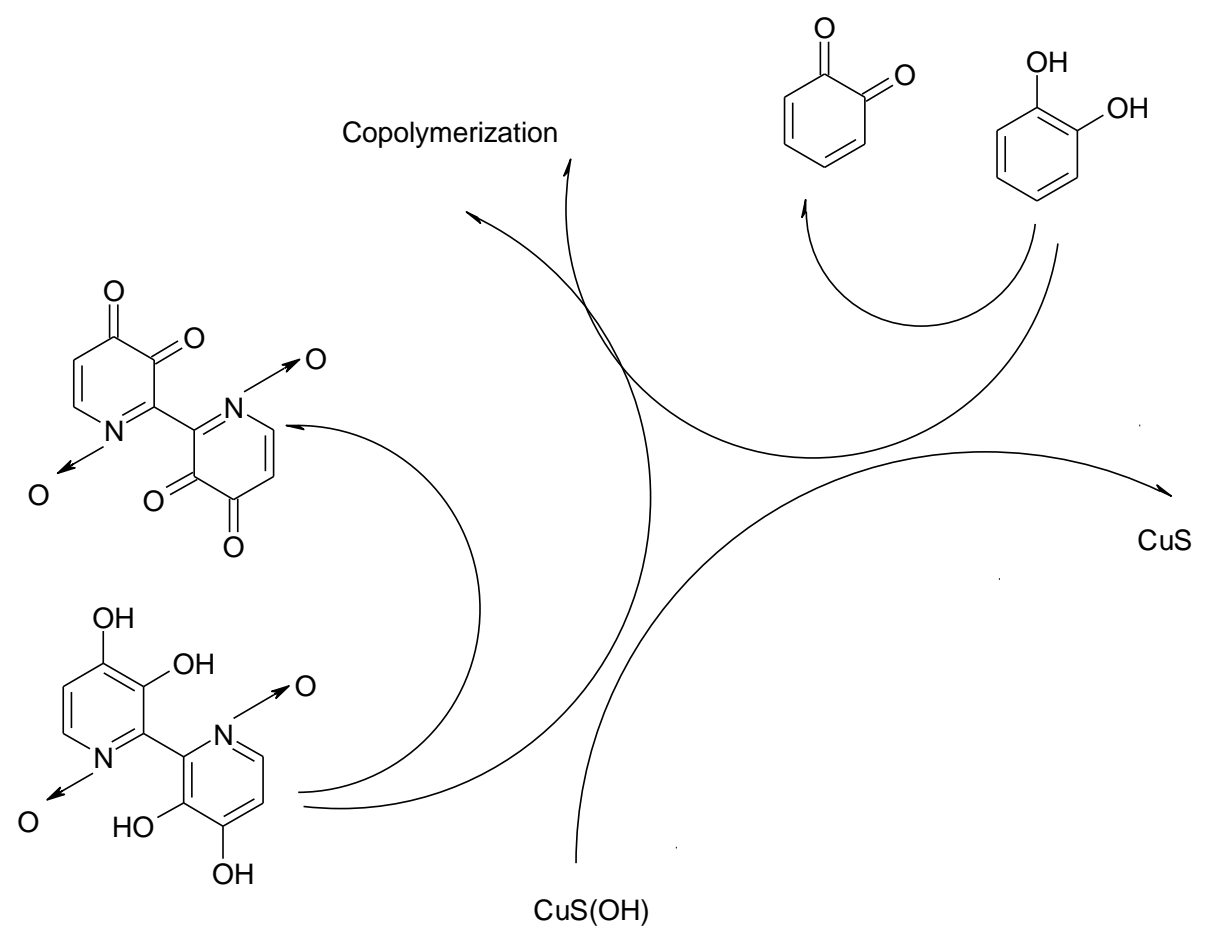

Figure 2. The scheme of the electroanalytical (electrosynthetical) process.

Therefore, in order to describe the electroanalytical process, we introduce three variables:

$\mathrm{o}$ - the orellanine concentration in the pre-surface layer;

$\mathrm{h}$ - hydroquinone concentration in the pre-surface layer;

c - copper sulfohydroxide surface coverage degree.

In order to simplify the modeling, we assume that:

- the background electrolyte is taken in excess so that we can neglect the migration flow and the oxidizing dopant oxidation change;

- the reactor is intensively stirred so that we may neglect the convection flow;

- the pre-surface layer concentration profile is linear, and its thickness is constant, equal to $\delta$.

It is possible to prove that the differential equations' set describing the system may be described as:

$\left\{\begin{array}{l}\frac{d o}{d t}=\frac{2}{\delta}\left(\frac{o}{\delta}\left(o_{0}-o\right)-r_{21}-r_{p}\right) \\ \frac{d h}{d t}=\frac{2}{\delta}\left(\frac{H}{\delta}\left(h_{0}-o\right)-r_{22}-r_{p}\right) \\ \frac{d c}{d t}=\frac{1}{C}\left(r_{1}-r_{21}-r_{22}-r_{p}\right)\end{array}\right.$

Herein, $\mathrm{O}$ and $\mathrm{H}$ are diffusion coefficients, $\mathrm{c}_{0}$ and $\mathrm{h} 0$ are the bulk concentrations of both of the compounds, $\mathrm{C}$ is the copper sulfohydroxide maximal surface coverage degree, and the parameters $r$ are correspondent reaction rates, calculated as is the copper ions bulk concentration, $\mathrm{M}$ is the complex compound maximal surface concentration, and the parameters $\mathrm{r}$ are the correspondent reaction rates, calculated as $(3-6)$ : 


$$
\begin{aligned}
& r_{1}=k_{1}(1-c) \exp \left(\frac{F \varphi_{0}}{R T}\right) \\
& r_{21}=k_{21} o c^{4} \exp (-a o) \\
& r_{22}=k_{22} h c^{2} \quad(5) \\
& r_{p}=k_{p} o^{x} h^{y} c^{z} \exp (-a o)
\end{aligned}
$$

Herein, the parameters $\mathrm{k}$ are the correspondent reaction rate constants, $\mathrm{x}, \mathrm{y}$, and $\mathrm{z}$ are polymerization reaction orders, a is the parameter relating the DEL capacitance with the complex formation, $\mathrm{F}$ is the Faraday number, $\varphi_{0}$ is the potential slope in DEL, related to the zero-charge potential, $\mathrm{R}$ is the universal gas constant, and $\mathrm{T}$ is the absolute temperature of the solution.

As it is possible to observe, the orellanine assisted electrooxidation and electropolymerization is more dynamic and affects the DEL more than the same process the simple hydroquinone, which contributes to the system's behavior. Nevertheless, the electrochemical process is efficient from both an electroanalytical and electrosynthetical point of view, as shown below.

\section{Results and Discussion}

In order to describe the electroanalytical process with orellaninee and hydroquinone nano-CuS-assisted electrochemical determination and electropolymerization, we analyze the equation-set (2) and algebraic relations $(3-6)$ by means of linear stability theory. The steadystate Jacobian matrix members for this case will be exposed as :

$\left(\begin{array}{lll}a_{11} & a_{12} & a_{13} \\ a_{21} & a_{22} & a_{23} \\ a_{31} & a_{32} & a_{33}\end{array}\right)$

in which:

$$
\begin{aligned}
& a_{11}=\frac{2}{\delta}\left(-\frac{o}{\delta}-k_{21} c^{4} \exp (-a o)+a k_{21} o c^{4} \exp (-a o)-x k_{p} o^{x-1} h^{y} c^{z} \exp (-a o)+\right. \\
& \left.a k_{p} o^{x} h^{y} c^{z} \exp (-a o)\right)(8) \\
& a_{12}=\frac{2}{\delta}\left(-y k_{p} o^{x} h^{y-1} c^{z} \exp (-a o)\right)(9) \\
& a_{13}=\frac{2}{\delta}\left(-4 k_{21} o c^{3} \exp (-a o)-z k_{p} o^{x} h^{y} c^{z-1} \exp (-a o)\right) \quad(10) \\
& a_{21}=\frac{2}{\delta}\left(-x k_{p} o^{x-1} h^{y} c^{z} \exp (-a o)+a k_{p} o^{x} h^{y} c^{z} \exp (-a o)\right)(11) \\
& a_{22}=\frac{2}{\delta}\left(-\frac{H}{\delta}-k_{22} c^{2}-y k_{p} o^{x} h^{y-1} c^{z} \exp (-a o)\right)(12) \\
& a_{23}=\frac{2}{\delta}\left(-2 k_{22} h c-z k_{p} o^{x} h^{y} c^{z-1} \exp (-a o)\right)(13) \\
& a_{31}=\frac{1}{c}\left(-k_{21} c^{4} \exp (-a o)+a k_{21} o c^{4} \exp (-a o)-x k_{p} o^{x-1} h^{y} c^{z} \exp (-a o)+\right. \\
& \left.a k_{p} o^{x} h^{y} c^{z} \exp (-a o)\right) \quad(14) \\
& a_{32}=\frac{1}{c}\left(-k_{22} c^{2}-y k_{p} o^{x} h^{y-1} c^{z} \exp (-a o)\right) \quad(15) \\
& a_{33}=\frac{1}{c}\left(-k_{1} \exp \left(\frac{F \varphi_{0}}{R T}\right)+j k_{1}(1-c) \exp \left(\frac{F \varphi_{0}}{R T}\right)-4 k_{21} o c^{3} \exp (-a o)-2 k_{22} h c-\right. \\
& \left.z k_{p} o^{x} h^{y} c^{z-1} \exp (-a o)\right) \quad(16)
\end{aligned}
$$

Taking into account the elements (8), (12) and (16), the oscillatory behavior may appear in this system. The main Hopf bifurcation condition is realized if the main diagonal contains positive elements related to the positive callback, and the Jacobian main diagonal possesses these addendums. 
Besides the addendum $j k_{1}(1-c) \exp \left(\frac{F \varphi_{0}}{R T}\right)>0$, if $\mathrm{j}>0$, describing the DEL influences of the electrochemical stage, related to the cyclic changes of surface impedance and conductivity, characteristic to more simple cases [27-28], the elements $a k_{21} o c^{4} \exp (-a o)>$ 0 and $a k_{p} o^{x} h^{y} c^{z} \exp (-a o)>0$ if $\mathrm{a}>0$, related to the orellanine oxidation and electropolymerization influence to DEL ionic force and, consequently, conductivity and impedance. The oscillations are expected to be frequent and of small amplitude, realizing beyond the detection limit.

It is important to mention that here the oscillatory behavior has become more probable than in the simplest case due to the ionic transformation of only one of two monomers. If both of the monomers are capable of manifesting a significant impact onDEL structure during their copolymerization, the oscillatory behavior will become even more probable in an analogous manner.

In order to investigate the steady-state stability, we apply the Routh-Hurwitz criterion to the equation-set (2). Avoiding the cumbersome expression, we introduce new variables, rewriting the determinant as (17):

$\frac{4}{\delta^{2} C}\left[\begin{array}{ccc}-\kappa-\Xi-\Lambda & -T & -\Pi-\Sigma \\ -\Xi & -\xi-H-T & -\Upsilon-\Sigma \\ -\Xi-\Lambda & -H-T & -\Omega-\Pi-\Upsilon-\Sigma\end{array}\right]$

Opening the brackets and applying the condition Det $\mathrm{J}<0$, salient from the criterion, we obtain the steady-state stability requirement, exposed as (18):

$-\kappa(\xi \Omega+\xi \Pi+\xi \Upsilon+\xi \Sigma+H \Omega+H \Pi+T \Omega+T \Pi)-\Xi(\xi \Omega+\xi \Upsilon+H \Omega+H \Pi)-\Lambda(\xi \Omega+$ $\xi \Upsilon+H \Omega+T \Omega+T \Upsilon-H \Sigma)<0$

This requirement is certainly satisfied if the above mentioned DEL influences are fragile and, consequently, may be easily neglected. If so, all of the variables of the inequation (18) will be positive, driving the left side of the inequation towards more negative values, stabilizing the system. Therefore, steady-state stability is easily formed.

As most of the elements contain the diffusion variables $\kappa$ (orellanine diffusion variable) and $\xi$ (hydroquinone diffusion variable), the system is diffusion-controlled.

As no side reaction compromising the analytes and modifier stability is observed in this system, the stable steady-state is both electroanalytical and electrosynthetically efficient, providing easy formation of linear dependence between the analytes' concentration and current and, if used electrosynthetically, well-developed copolymer formation, such as in similar systems [27-28].

As for the monotonic instability, corresponding to the detection limit, it delimits the margin between the stable steady-states and unstable states, being correspondent to the bifurcation of stable "node" and unstable "saddle". Its condition is Det $\mathrm{J}=0$, or (19):

$-\kappa(\xi \Omega+\xi \Pi+\xi \Upsilon+\xi \Sigma+H \Omega+H \Pi+T \Omega+T \Pi)-\Xi(\xi \Omega+\xi \Upsilon+H \Omega+H \Pi)-\Lambda(\xi \Omega+$ $\xi \Upsilon+H \Omega+T \Omega+T \Upsilon-H \Sigma)<0$

In the case of electropolymerization and simultaneous electrochemical determination of orellanine with another compound with oxidized or ionic pyridinic nitrogen, both of the compounds will affect the DEL ionic force, augmenting, therefore, the probability of the oscillatory behavior. This case will be described in one of our next works. 


\section{Conclusions}

From the theoretical model evaluation of nano-CuS-assisted orellanine and hydroquinone simultaneous detection, it has been possible to conclude that it is an efficient process from both electroanalytical and electrosynthetical points of view, providing an economical and green conducting polymer synthesis. The linear dependence between the analytes' concentrations and electrochemical parameters is easy to form in an efficient diffusion-controlled system. For its turn, the oscillatory behavior is expected to be probable due to the double electric layer structure changes during the process on all of the stages. The probability of the oscillatory behavior and the oscillation amplitude will be highly dependent on the solution background electrolyte composition.

\section{Funding}

This research received no external funding.

\section{Acknowledgments}

This research has no acknowledgment.

\section{Conflicts of Interest}

The authors declare no conflict of interest.

\section{References}

1. Marchelek-Myśliwiec, M.; Wilk, A.; Turoń-Skrzypińska, A.; Nowosiad-Magda, M.; Dutkiewicz, G.; Dołęgowska, B.; Stępniewska, J.; Ciechanowski, K.; Wiszniewska, B. Acute kidney injury related to wild mushrooms intoxication.J. Educ. Health. Sport. 2020, 10, 42-46, https://doi.org/10.12775/JEHS.2020.10.01.005.

2. Dinis-Oliveira, R.J.; Soares, M.; Rocha-Pereira, C.; Carvalho, F. Human and experimental toxicology of orellaninee. Human \& Experimental Toxicology 2015, 35, 10161029,https://doi.org/10.1177/0960327115613845.

3. Beaumier, M.; Rioult, J.-P.; Georges, M.; Brocheriou, I.; Lobbedez, T.; Lanot, A. Mushroom Poisoning Presenting WithAcute Kidney Injury and Elevated Transaminases. Kidney International Reports 2019, 4, 877-881,https://doi.org/10.1016/j.ekir.2019.02.016.

4. Khovpachev, A.A.; Basharin, V.A.; Chepur, S.V. The Modern State of Art of The High Mushroom Toxins. Nitrogen-Containing Compounds. Uspekhi. Sovr. Biol. 2020, 140, 378-394, in Russian, https://doi.org/10.31857/S0042132420040092.

5. Xin, Y.; Wang, N.; Wang, C.; Gao, W.; Chen, M.; Liu, N.; Duan, J.; Hou, B. Electrochemical detection of hydroquinone and catechol with covalent organic framework modified carbon paste electrode. Journal of Electroanalytical Chemistry 2020, 877, https://doi.org/10.1016/j.jelechem.2020.114530.

6. Karthika, A.; Ramasamy Raja, V.; Karuppasamy, P.; Suganthi, A.; Rajarajan, M. A novel electrochemical sensor for determination of hydroquinone in water using FeWO4/SnO2 nanocomposite immobilized modified glassy carbon electrode. Arabian Journal of Chemistry 2020, 13, 4065-4081, https://doi.org/10.1016/j.arabjc.2019.06.008.

7. Kumar, Y.; Vashistha, V.K.; Das, D.K. Synthesis of Perovskite-type $\mathrm{NdFeO}_{3}$ nanoparticles and used as electrochemical sensor for detection of paracetamol. Lett. Appl. Nanobiosci. 2020, 9, 866-869, https://doi.org/10.33263/LIANBS91.866869.

8. Sá, A.C.d.; Barbosa, S.C.; Raymundo-Pereira, P.A.; Wilson, D.; Shimizu, F.M.; Raposo, M.; Oliveira Jr, O.N. Flexible Carbon Electrodes for Electrochemical Detection of Bisphenol-A, Hydroquinone and Catechol in Water Samples. Chemosensors 2020, 8,https://doi.org/10.3390/chemosensors8040103.

9. Koçak, Ç.C.; Koçak, S. Enhanced Electrochemical Determination of Catechol and Hydroquinone Based on Pd Nanoparticles/Poly(Taurine) Modified Glassy Carbon Electrode. Electroanalysis 2020, 32, 358-366, https://doi.org/10.1002/elan.201900500.

10. Mashhadizadeh, M.H.; Kalantarian, S.M.; Azhdeh, A. A Novel Electrochemical Sensor for Simultaneous Determination of Hydroquinone, Catechol, and Resorcinol using a Carbon Paste Electrode modified by Zn- 
MOF, Nitrogen-doped Graphite, and AuNPs. Electroanalysis 2020,32, https://doi.org/10.1002/elan.202060326.

11. Manjunatha, J. Poly(Adenine) Modified Graphene-Based Voltammetric Sensor for the Electrochemical Determination of Catechol, Hydroquinone and Resorcinol.The Open Chemical Engineering Journal 2020, 14, 52-62, http://dx.doi.org/10.2174/1874123102014010052.

12. Ahmad, K.; Kumar, P.; Mobin, S.M. A highly sensitive and selective hydroquinone sensor based on a newly designed $\mathrm{N}-\mathrm{rGO} / \mathrm{SrZrO} 3 \quad$ composite. Nanoscale Advances 2020, 2, 502-511, https://doi.org/10.1039/C9NA00573K.

13. Romero-Montero, A.; del Valle, L.J.; Puiggalí, J.; Montiel, C.; García-Arrazola, R.; Gimeno, M. Poly(gallic acid)-coated polycaprolactone inhibits oxidative stress in epithelial cells. Materials Science and Engineering: C 2020, 115, https://doi.org/10.1016/j.msec.2020.111154.

14. Zamudio-Cuevas, Y.; Andonegui-Elguera, M.A.; Aparicio-Juárez, A.; Aguillón-Solís, E.; Martínez-Flores, K.; Ruvalcaba-Paredes, E.; Velasquillo-Martínez, C.; Ibarra, C.; Martínez-López, V.; Gutiérrez, M.; GarcíaArrazola, R.; Hernández-Valencia, C.G.; Romero-Montero, A.; Hernández-Valdepeña, M.A.; Gimeno, M.; Sánchez-Sánchez, R. The enzymatic poly(gallic acid) reduces pro-inflammatory cytokines in vitro, a potential application in inflammatory diseases. Inflammation 2020, https://doi.org/10.1007/s10753-02001319-5.

15. Yang, P.; Zhang, S.; Chen, X.; Liu, X.; Wang, Z.; Li, Y. Recent developments in polydopamine fluorescent nanomaterials. Materials Horizons 2020, 7, 746-761, https://doi.org/10.1039/c9mh01197h.

16. Zmerli, I.; Michel, J.-P.; Makky, A. Bioinspired polydopamine nanoparticles: synthesis, nanomechanical properties, and efficient PEGylation strategy. Journal of Materials Chemistry B 2020, 8, 4489-4504, https://doi.org/10.1039/c9tb02769f.

17. Murari, G.; Bock, N.; Zhou, H.; Yang, L.; Liew, T.; Fox, K.; Tran, P.A. Effects of polydopamine coatings on nucleation modes of surface mineralization from simulated body fluid. Scientific Reports 2020, 10,https://doi.org/10.1038/s41598-020-71900-3.

18. Li, H.; Xi, J.; Donaghue, A.G.; Keum, J.; Zhao, Y.; An, K.; McKenzie, E.R.; Ren, F. Synthesis and catalytic performance of polydopamine supported metal nanoparticles. Scientific Reports 2020, 10,https://doi.org/10.1038/s41598-020-67458-9.

19. Wang, Z.; Zou, Y.; Li, Y.; Cheng, Y. Metal-Containing Polydopamine Nanomaterials: Catalysis, Energy, and Theranostics. Small 2020, 16,https://doi.org/10.1002/smll.201907042.

20. Jin, A.; Wang, Y.; Lin, K.; Jiang, L. Nanoparticles modified by polydopamine: Working as "drug” carriers. Bioactive Materials 2020, 5, 522-541,https://doi.org/10.1016/j.bioactmat.2020.04.003.

21. Gargioni, C.; Borzenkov, M.; D’Alfonso, L.; Sperandeo, P.; Polissi, A.; Cucca, L.; Dacarro, G.; Grisoli, P.; Pallavicini, P.; D’Agostino, A.; Taglietti, A. Self-Assembled Monolayers of Copper Sulfide Nanoparticles on Glass as Antibacterial Coatings. Nanomaterials 2020, 10,https://doi.org/10.3390/nano10020352.

22. Rajaram, R.; Kiruba, M.; Suresh, C.; Mathiyarasu, J.; Kumaran, S.; Kumaresan, R. Amperometric determination of Myo-inositol using a glassy carbon electrode modified with nanostructured copper sulfide. Microchimica Acta 2020, 187, https://doi.org/10.1007/s00604-020-04300-z.

23. Alshahrani, L.A.; Miao, L.; Zhang, Y.; Cheng, S.; Sathishkumar, P.; Saravanakumar, B.; Nan, J.; Gu, F.L. 3D-Flower-Like Copper Sulfide Nanoflake-Decorated Carbon Nanofragments-Modified Glassy Carbon Electrodes for Simultaneous Electrocatalytic Sensing of Co-existing Hydroquinone and Catechol. Sensors 2019, 19,https://doi.org/10.3390/s19102289.

24. Malakootian, M.; Hamzeh, S.; Mahmoudi-Moghaddam, H. A Noverl Electrochemical Sensor Based on $\mathrm{FeNi}_{3} / \mathrm{CuS} / \mathrm{BiOCl}$ Modified Carbon Paste Electrode for Determination of Bisphenol A.Electroanalysis 2020, 32, https://doi.org/10.1002/elan.202060205.

25. Das, I.; Goel, N.; Agrawal, N.R.; Gupta, S.K. Growth Patterns of Dendrimers and Electric Potential Oscillations during Electropolymerization of Pyrrole using Mono- and Mixed Surfactants. The Journal of Physical Chemistry B 2010, 114, 12888-12896,https://doi.org/10.1021/jp105183q.

26. Das, I.; Goel, N.; Gupta, S.K.; Agrawal, N.R. Electropolymerization of pyrrole: Dendrimers, nano-sized patterns and oscillations in potential in presence of aromatic and aliphatic surfactants. Journal of Electroanalytical Chemistry 2012, 670, 1-10,https://doi.org/10.1016/j.jelechem.2012.01.023.

27. Tkach, V.; Kushnir, M.; Ivanushko, Y.; de Oliveira, S.; Reis, L.; Yagodynets’', P.; Kormosh, Z. The theoretical description for neotame electrochemical determination, assisted by vanadium oxyhydroxide composite with a squarainic dye. Appl. J. Env. Eng. Sci. 2020, 6, 109-115.

28. Tkach, V.V.; Kushnir, M.V.; de Oliveira, S.C.; Ivanushko, Y.G.; Velyka, A.V.; Molodianu, A.F.; Yagodynets, P.I.; Kormosh, Z.O.; Reis, L.V.d.; Luganska, O.V.; Palamarek, K.V.; Bredikhina, Y.L.Electrochemical Determination of Sudan Dyes and Two Manners to Realize it: a Theoretical Investigation. Lett. Appl. NanoBioSci. 2020, 9, 1451-1458, https://doi.org/10.33263/LIANBS94.14511458. 